\title{
Exact Vacuum Solutions to the Einstein Equation
}

\author{
Ying-Qiu Gu* \\ School of Mathematical Science, Fudan University, Shanghai 200433, China
}

\begin{abstract}
In this paper, we present a framework for getting a series of exact vacuum solutions to the Einstein equation. This procedure of resolution is based on a canonical form of the metric. According to this procedure, the Einstein equation can be reduced to some 2-dimensional Laplace-like equations or rotation and divergence equations, which are much convenient for the resolution.
\end{abstract}

Keywords: Einstein equation, exact vacuum solution, canonical metric, black hole

2000 MR Subject Classification: 83C05, 83C15, 83C57

\section{Introduction}

The Einstein equation

$$
G_{\mu \nu} \stackrel{\text { def }}{=} R_{\mu \nu}-\frac{1}{2} g_{\mu \nu} R=\kappa T_{\mu \nu}
$$

is a highly nonlinear system of partial differential equations satisfied by the metric $g_{\mu \nu}$, where $R_{\mu \nu}$ stands for the Ricci tensor and $R$ the scalar curvature of the spacetime manifold defined respectively by

$$
R_{\mu \nu}=\partial_{\alpha} \Gamma_{\mu \nu}^{\alpha}-\partial_{\mu} \Gamma_{\nu \alpha}^{\alpha}+\Gamma_{\mu \nu}^{\alpha} \Gamma_{\alpha \beta}^{\beta}-\Gamma_{\mu \beta}^{\alpha} \Gamma_{\nu \alpha}^{\beta},
$$

and

$$
R=g^{\mu \nu} R_{\mu \nu}
$$

*email: yqgu@luody.com.cn 
in which $\Gamma_{\mu \nu}^{\alpha}$ is the Christoffel symbol given by

$$
\Gamma_{\mu \nu}^{\alpha}=\frac{1}{2} g^{\alpha \beta}\left(\partial_{\mu} g_{\beta \nu}+\partial_{\nu} g_{\mu \beta}-\partial_{\beta} g_{\mu \nu}\right)
$$

$T_{\mu \nu}$ is the energy-momentum tensor of matter, and the Greek indexes go from 0 to 3 or from $t, z, x$ to $y$. In the vacuum domain we have

$$
T_{\mu \nu} \equiv 0
$$

To get the exact vacuum solution of the Einstein equation is an arduous work $[1,2]$. The conventional method of resolution is to analyze the symmetry of the metric, i.e. analyze the Killing vectors of the spacetime. Almost all the well-known solutions such as the Friedmann-Robertson-Walker metric, Bianchi universe, Lemaitre-de Sitter universe, as well as Schwarzschild metric and Kerr metric, Taub-NUT solution[2, 3, 4], are based on the symmetry of spacetime.

Obviously, a good choice for the coordinate system of the spacetime manifold should be very helpful for analyzing the Einstein equation. The traditional choices are the Gaussian coordinate system or the harmonic coordinate system[5]. The former gives locally the following metric

$$
g_{\mu \nu}=\left(\begin{array}{cccc}
1 & 0 & 0 & 0 \\
0 & g_{11} & g_{12} & g_{13} \\
0 & g_{21} & g_{22} & g_{23} \\
0 & g_{31} & g_{32} & g_{33}
\end{array}\right),
$$

while, the latter satisfies the following coordinate condition

$$
g^{\alpha \beta} \Gamma_{\alpha \beta}^{\mu}=0
$$

However (1.6) and (1.7) are more convenient only for some theoretical analysis rather than for practical resolution.

Fortunately, the problem can be much simplified in a specially chosen coordinate system. Under some natural assumptions we have found that the metric can be transformed into the following canonical form

$$
g_{\mu \nu}=\left(\begin{array}{cccc}
u & v & p & q \\
v & 0 & 0 & 0 \\
p & 0 & -a & 0 \\
q & 0 & 0 & -b
\end{array}\right)
$$


with

$$
\operatorname{det}\left(g_{\mu \nu}\right)=-v^{2} a b
$$

and the inverse

$$
g^{\mu \nu}=\left(\begin{array}{cccc}
0 & v^{-1} & 0 & 0 \\
v^{-1} & -\frac{u a b+p^{2} b+q^{2} a}{v^{2} a b} & \frac{p}{v a} & \frac{q}{v b} \\
0 & \frac{p}{v a} & -a^{-1} & 0 \\
0 & \frac{q}{v b} & 0 & -b^{-1}
\end{array}\right),
$$

where $u, v, p, q, a, b$ are smooth functions of the coordinates $(t, z, x, y)$ (See [6] for the proof of this canonical form).

The canonical form (1.8) is an Alibaba's conjuration for solving the Einstein equation. In what follows, we will show how to use this metric to solve the vacuum Einstein equation.

\section{Procedure of Resolution}

For the canonical metric (1.8), computing the Einstein tensor $G_{\mu \nu}$, we get

$$
G_{z z}=-\frac{1}{2}\left[\left(\frac{\partial b}{b \partial z}+\frac{\partial a}{a \partial z}\right) \frac{\partial v}{v \partial z}+\frac{1}{2}\left(\frac{\partial a}{a \partial z}\right)^{2}+\frac{1}{2}\left(\frac{\partial b}{b \partial z}\right)^{2}-\frac{\partial^{2} a}{a \partial z^{2}}-\frac{\partial^{2} b}{b \partial z^{2}}\right] .
$$

If $\partial_{z}(a b) \neq 0$, solving $G_{z z}=0$ with respect to $v$, we get

$$
v=V \exp \left[\int\left(\frac{\partial^{2} a}{a \partial z^{2}}+\frac{\partial^{2} b}{b \partial z^{2}}-\frac{1}{2}\left(\frac{\partial a}{a \partial z}\right)^{2}-\frac{1}{2}\left(\frac{\partial b}{b \partial z}\right)^{2}\right)\left(\frac{\partial b}{b \partial z}+\frac{\partial a}{a \partial z}\right)^{-1} d z\right],
$$

where $V=V(t, x, y)$ is a function to be determined.

In order that the following recursive procedure goes on, for the exact solutions, we must adopt an ansatz for $a$ and $b$ to make (2.2) integrable. Different ansatz leads to different family of solutions. In what follows we take the separating variable ansatz as a representative example to show how the procedure works, that is, we assume

$$
a=L(t, x, y) K(t, z)^{2}, \quad b=N(t, x, y) K(t, z)^{2},
$$

where $\partial_{z} K \neq 0$.

Substituting (2.3) into (2.2), it is easy to see that

$$
v=V \partial_{z} K
$$

Using (2.3) and (2.4), we have

$$
G_{z x}=-\frac{1}{2 V}\left(\frac{\partial_{z}^{2} p}{\partial_{z} K}-\frac{\partial_{z}^{2} K \partial_{z} p}{\left(\partial_{z} K\right)^{2}}-2 p \frac{\partial_{z} K}{K^{2}}+2 \partial_{x} V \frac{\partial_{z} K}{K}\right)
$$


Solving $G_{z x}=0$ with respect to $p$, we get

$$
p=P K^{2}+\frac{\partial V}{\partial x} K+\frac{A}{K},
$$

where $P, A$ are functions of $t, x, y$ to be determined.

Similarly, we have

$$
G_{z y}=-\frac{1}{2 V}\left(\frac{\partial_{z}^{2} q}{\partial_{z} K}-\frac{\partial_{z}^{2} K \partial_{z} q}{\left(\partial_{z} K\right)^{2}}-2 q \frac{\partial_{z} K}{K^{2}}+2 \partial_{y} V \frac{\partial_{z} K}{K}\right)
$$

Solving $G_{z y}=0$ with respect to $q$, we get

$$
q=-Q K^{2}+\frac{\partial V}{\partial y} K+\frac{B}{K}
$$

where $Q, B$ are functions of $t, x, y$ to be determined.

Substituting (2.3), (2.4), (2.6) and (2.8) into $G_{x y}=0$, we get

$$
\partial_{x} Q-\partial_{y} P=-P \frac{\partial_{y} L}{L}+Q \frac{\partial_{x} N}{N}
$$

and

$$
A=B=0 .
$$

By $G_{x x}=0$ or $G_{y y}=0$, we obtain

$$
\frac{\partial_{x} P}{L}+\frac{\partial_{y} Q}{N}-\frac{P}{2 L}\left(\frac{\partial_{x} L}{L}+\frac{\partial_{x} N}{N}\right)-\frac{Q}{2 N}\left(\frac{\partial_{y} L}{L}+\frac{\partial_{y} N}{N}\right)=-\frac{1}{2}\left(\frac{\partial_{t} L}{L}-\frac{\partial_{t} N}{N}\right) .
$$

Therefore, taking $(P, Q)$ as a vector in subspace $(x, y),(2.9)$ and $(2.11)$ provide the corresponding covariant rotation and divergence equations respectively.

By $G_{t z}=0$, we get

$$
u=-\left(\frac{P^{2}}{L}+\frac{Q^{2}}{N}\right) K^{2}+2 \partial_{t}(V K)+W V K+U+\frac{\alpha}{V K},
$$

where $\alpha$ is a function independent of $z$ to be determined. $U(t, x, y)$ and $W(t, x, y)$ satisfy

$$
\Delta V=\frac{U+|\nabla V|^{2}}{V}-S V
$$

with

$$
S=\frac{1}{4}\left(\frac{\left(\partial_{x} N\right)^{2}}{L N^{2}}+\frac{\left(\partial_{y} L\right)^{2}}{L^{2} N}+\frac{\partial_{x} L \partial_{x} N}{L^{2} N}+\frac{\partial_{y} L \partial_{y} N}{L N^{2}}-\frac{2}{L N}\left(\partial_{x}^{2} N+\partial_{y}^{2} L\right)\right),
$$

and

$$
\begin{aligned}
\frac{\partial_{x} P}{L}-\frac{\partial_{y} Q}{N} & -\frac{P}{2 L}\left(\frac{\partial_{x} L}{L}-\frac{\partial_{x} N}{N}\right)-\frac{Q}{2 N}\left(\frac{\partial_{y} L}{L}-\frac{\partial_{y} N}{N}\right)+\frac{1}{2}\left(\frac{\partial_{t} L}{L}+\frac{\partial_{t} N}{N}\right) \\
& =W+\frac{2}{V}\left(\partial_{t} V+\frac{1}{L} P \partial_{x} V-\frac{1}{N} Q \partial_{y} V\right),
\end{aligned}
$$


in which the covariant Laplace and gradient operators of a scalar in the subspace $(x, y)$ are defined respectively by

$$
\begin{aligned}
\Delta & =\frac{1}{L} \partial_{x}^{2}+\frac{1}{N} \partial_{y}^{2}+\frac{1}{2}\left(-\frac{\partial_{x} L}{L^{2}}+\frac{\partial_{x} N}{L N}\right) \partial_{x}+\frac{1}{2}\left(\frac{\partial_{y} L}{L N}-\frac{\partial_{y} N}{N^{2}}\right) \partial_{y} \\
\nabla & =\left(\frac{1}{\sqrt{L}} \partial_{x}, \frac{1}{\sqrt{N}} \partial_{y}\right) .
\end{aligned}
$$

By $G_{t x}=G_{t y}=0$, we find $\alpha=\alpha(t)$. We should point out that, $G_{t x}=G_{t y}=0$ also provide two linear Laplace-like equations of $P$ and $Q$, which are consequences of (2.9) and (2.11).

For $G_{t t}=0$, a direct calculation leads to

$$
\begin{aligned}
\Delta U & =-\frac{3 W \alpha+2 \partial_{t} \alpha}{V^{2}} \\
\Delta W & =-\frac{2}{V^{2}}\left(W U+\partial_{t} U+\frac{1}{L} P \partial_{x} U-\frac{1}{N} Q \partial_{y} U\right) .
\end{aligned}
$$

Thus, we can take $U, V, W, P, Q, \alpha$ as unknown functions satisfying the almost linear partial differential equations (2.18), (2.13), (2.19), (2.9), (2.11) and (2.15), while, take $K(t, z), L(t, x, y), N(t, x, y)$ as functions determined by coordinates, boundary conditions and initial conditions. Any one of the equations (2.18), (2.13) and (2.19) is quite similar to the Ernst equation[7, 8].

Furthermore, we can simplify the metric by setting $r=K$ as new coordinate to replace $z$ as follows. The line element of the spacetime is then given by

$$
\begin{aligned}
d s^{2} & =u d t^{2}+2 d t(v d z+p d x+q d y)-\left(a d x^{2}+b d y^{2}\right), \\
& =\left(u-2 V \partial_{t} K\right) d t^{2}+2 V d t d K+2 d t(p d x+q d y)-\left(a d x^{2}+b d y^{2}\right), \\
& =\bar{u} d t^{2}+2 V d t d r+2 d t(p d x+q d y)-\left(a d x^{2}+b d y^{2}\right),
\end{aligned}
$$

where

$$
\bar{u}=-\left(\frac{P^{2}}{L}+\frac{Q^{2}}{N}\right) r^{2}+\left(2 \partial_{t} V+W V\right) r+U+\frac{\alpha}{V r} .
$$

Thus, in the new coordinate system $(t, r, x, y)$, the metric becomes

$$
g_{\mu \nu}=\left(\begin{array}{cccc}
\bar{u} & V & P r^{2}+\partial_{x} V r & -Q r^{2}+\partial_{y} V r \\
V & 0 & 0 & 0 \\
P r^{2}+\partial_{x} V r & 0 & -L r^{2} & 0 \\
-Q r^{2}+\partial_{y} V r & 0 & 0 & -N r^{2}
\end{array}\right) .
$$

Similarly, if we take $\rho=V K$ as new coordinate to replace $z$, then in the new 
coordinate system $(t, \rho, x, y)$, the metric becomes

$$
g_{\mu \nu}=\left(\begin{array}{cccc}
-\left(\frac{P^{2}}{L}+\frac{Q^{2}}{N}\right) \frac{\rho^{2}}{V^{2}}+W \rho+U+\frac{\alpha}{\rho} & 1 & \frac{P}{V^{2}} \rho^{2} & -\frac{Q}{V^{2}} \rho^{2} \\
1 & 0 & 0 & 0 \\
\frac{P}{V^{2}} \rho^{2} & 0 & -\frac{L}{V^{2}} \rho^{2} & 0 \\
-\frac{Q}{V^{2}} \rho^{2} & 0 & 0 & -\frac{N}{V^{2}} \rho^{2}
\end{array}\right) .
$$

The above procedure can provide a family of solutions depending on 4 coordinates, which is relatively complicated. However, for some special cases including more symmetries, the procedure can be much simpler. For instance, if we consider the static case with the following ansatz

$$
a=b=a(x, y), \quad p=q=0, \quad u=u(x, y), \quad v=v(x, y)
$$

then the solutions can be explicitly obtained. In fact, for this case, $G_{t x}, G_{t y}, G_{z z}, G_{z x}$ and $G_{z y}$ vanish automatically. By $G_{x x}+G_{y y}=0$, we get

$$
\left(\partial_{x}^{2}+\partial_{y}^{2}\right) v=0
$$

that is to say, $v(x, y)$ is a 2-dimensional harmonic function, which can be easily generated by means of complex analytic function $f(x+y i)$.

Moreover, by $G_{x x}-G_{y y}=G_{x y}=G_{t t}=0$ and (2.25), we get

$$
\begin{aligned}
\left(\partial_{x}^{2}+\partial_{y}^{2}\right) a & =\frac{1}{a}\left(\left|\partial_{x} a\right|^{2}+\left|\partial_{y} a\right|^{2}\right)+\frac{a}{2 v^{2}}\left(\left|\partial_{x} v\right|^{2}+\left|\partial_{y} v\right|^{2}\right), \\
\left(\partial_{x}^{2}+\partial_{y}^{2}\right) u & =\frac{1}{v}\left(\partial_{x} u \partial_{x} v+\partial_{y} u \partial_{y} v\right)-\frac{u}{v^{2}}\left(\left|\partial_{x} v\right|^{2}+\left|\partial_{y} v\right|^{2}\right) .
\end{aligned}
$$

The solution of $(2.26)$ consistent with $G_{x x}=G_{x y}=G_{y y}=0$ is given by

$$
a=\frac{\lambda}{\sqrt{v}}\left(\left|\partial_{x} v\right|^{2}+\left|\partial_{y} v\right|^{2}\right)
$$

where $\lambda$ is a constant.

(2.27) is a linear equation of $u$. Let $u=v w$, we get

$$
\left(\partial_{x}^{2}+\partial_{y}^{2}\right) w=-\frac{1}{v}\left(\partial_{x} w \partial_{x} v+\partial_{y} w \partial_{y} v\right)
$$

For any given $v$, the solution of (2.29) can be easily determined by an appropriate boundary condition. Here we provide the analytic solution related to $v$, which is given by

$$
u=v[m+n \ln v+(k+j \ln v) \bar{v}],
$$

where $j, k, m, n$ are constants, $\bar{v}$ is the conjugate harmonic function of $v$, namely,

$$
v=\Re(f(x+y i)), \quad \bar{v}=\Im(f(x+y i)),
$$




$$
v=\Im(f(x+y i)), \quad \bar{v}=\Re(f(x+y i)) .
$$

For other ansatz of the metric, the recursive resolution procedure is basically along the same line, that is

$$
G_{z z} \rightarrow\left(G_{z x}, G_{z y}\right) \rightarrow\left(G_{x y}, G_{x x}, G_{y y}, G_{t z}\right) \rightarrow\left(G_{t x}, G_{t y}\right) \rightarrow G_{t t}
$$

\section{$3 \quad$ Examples}

Example 1. For the case $(2.24) \sim(2.30)$, if we take the complex function as

$$
f=\frac{Z}{R}-\frac{R}{Z}
$$

where $Z=x+y i$ and $R$ is a real number, we have

$$
v=\Re(f)=\frac{x\left(x^{2}+y^{2}-R^{2}\right)}{R\left(x^{2}+y^{2}\right)}, \quad \bar{v}=\Im(f)=\frac{y\left(x^{2}+y^{2}+R^{2}\right)}{R\left(x^{2}+y^{2}\right)}
$$

Substituting (3.2) into (2.28) and (2.30), we get

$$
\begin{aligned}
& a=\frac{\lambda\left(x^{4}+2 x^{2} y^{2}+2 R^{2} x^{2}+y^{4}+R^{4}-2 R^{2} y^{2}\right)}{R \sqrt{x\left(x^{2}+y^{2}-R^{2}\right)\left(x^{2}+y^{2}\right)^{3} R}}, \\
& u=\frac{x\left(x^{2}+y^{2}-R^{2}\right)}{R\left(x^{2}+y^{2}\right)}\left(m+n \ln v+(k+j \ln v) \frac{y\left(x^{2}+y^{2}+R^{2}\right)}{R\left(x^{2}+y^{2}\right)}\right) .
\end{aligned}
$$

This is a more complicated version than that presented in [9].

Example 2. For the case $(2.24) \sim(2.30)$, if we take the complex function as

$$
f=\cosh \left(\frac{x+y i}{R}\right)
$$

where $R$ is a real number, then we have

$$
v=\Re(f)=\cosh \frac{x}{R} \cos \frac{y}{R}, \quad \bar{v}=\Im(f)=\sinh \frac{x}{R} \sin \frac{y}{R} .
$$

Substituting (3.6) into (2.28) and (2.30), we get

$$
\begin{aligned}
a & =\frac{\lambda}{R^{2}}\left(\cosh ^{2} \frac{x}{R}-\cos ^{2} \frac{y}{R}\right) \sqrt{\left(\cosh \frac{x}{R} \cos \frac{y}{R}\right)^{-1}}, \\
u & =\cosh \frac{x}{R} \cos \frac{y}{R}\left(m+n \ln v+(k+j \ln v)\left(\sinh \frac{x}{R} \sin \frac{y}{R}\right)\right) .
\end{aligned}
$$

Obviously, we can construct infinite solutions in this family by choosing different analytic function $f$. 
Example 3. For the static case of metric (2.22), if we take the following ansatz

$$
L=1, \quad N=\sin ^{2} x, \quad U=0,
$$

then $S=1$ in (2.13) and we can get

$$
W=0, \quad P=m \sin x, \quad Q=k \sin ^{2} x, \quad V=n \sin x \tan ^{\beta} \frac{x}{2},
$$

where $k, m, n, \beta$ are constants.

If we take $k=\alpha, m=\beta=0, n=1$ in (3.10), under a suitable coordinate transformation, from (3.9) and (3.10) we can get a clear and normal form of metric (2.22) as follows

$$
g_{\mu \nu}=\left(\begin{array}{cccc}
\frac{\alpha}{r \sin x} & \sin x & r \cos x & 0 \\
\sin x & -\frac{r}{r-R} \sin ^{2} x & 0 & \sqrt{\frac{r}{r-R}} r \sin ^{2} x \\
r \cos x & 0 & -r^{2} & 0 \\
0 & \sqrt{\frac{r}{r-R}} r \sin ^{2} x & 0 & -r^{2} \sin ^{2} x
\end{array}\right),
$$

where $\alpha, R$ are constants. In this case, the corresponding spacetime has a torus structure.

Example 4. For the dynamic case of metric (2.22), if we take the following ansatz

$$
L=1, \quad N=\sin ^{2} x, \quad P=Q=\partial_{y} V=0,
$$

then we can get

$$
\begin{aligned}
W & =-\frac{2}{f} f^{\prime}(t), \quad U=k f^{2}, \quad \alpha=m f^{3}, \\
V & =\frac{f \sin x}{2 \beta \sqrt{\beta n}}\left(\beta \tan ^{-\beta} \frac{x}{2}+k n \tan ^{\beta} \frac{x}{2}\right),
\end{aligned}
$$

or

$$
V=\frac{f \sin x}{2 \beta \sqrt{\beta n}}\left(n \beta \tan ^{-\beta} \frac{x}{2}+k \tan ^{\beta} \frac{x}{2}\right)
$$

where $k, m$ and $n \beta>0$ are constants, and $f(t)$ is an arbitrary function of $t$.

In particular, setting $k=1, \beta=\frac{1}{2}, n=2$ and

$$
f=1+\varepsilon \sin (\omega t)
$$

using (3.15) we get

$$
V=(1+\varepsilon \sin (\omega t)) \sin x\left(\sqrt{\tan \frac{x}{2}}+\sqrt{\tan ^{-1} \frac{x}{2}}\right),
$$


then metric (2.23) becomes

$$
g_{\mu \nu}=\operatorname{diag}\left\{\left(\begin{array}{cc}
f^{2}+\frac{m}{\rho} f^{3}-\frac{2}{f} f^{\prime} \rho & 1 \\
1 & 0
\end{array}\right),-\frac{\rho^{2}}{V^{2}},-\frac{\rho^{2}}{V^{2}} \sin ^{2} x\right\},
$$

where $\varepsilon$ is a constant, $(t, \rho, x, y)$ are the coordinates. This metric provides a rigorous model for the study of the behavior of gravitational waves.

Example 5. For the periodic case of metric (2.22), if we take the following ansatz

$$
L=1, \quad N=\sin ^{2} x, \quad V=1,
$$

then we can get

$$
\begin{gathered}
U=1, \quad W=0, \quad \alpha=-2 m, \\
P=f \cos (y-h), \quad Q=f \cos x \sin x \sin (y-h),
\end{gathered}
$$

where $m$ is a constant, and $f(t), h(t)$ are arbitrary functions of $t$. This metric provides a strange rotary gravity.

Setting $f=0$ and making a coordinate transformation

$$
t=\tau-r-2 m \ln (r-2 m), \quad x=\theta, \quad y=\varphi,
$$

metric (2.22) becomes the normal Schwarzschild metric in system $(\tau, r, \theta, \varphi)$ :

$$
g_{\mu \nu}=\operatorname{diag}\left\{1-\frac{2 m}{r},-\left(1-\frac{2 m}{r}\right)^{-1}, r^{2}, r^{2} \sin ^{2} \theta\right\} .
$$

Example 6. For metric (2.22), if we take $L=N=1$, then we have the following solutions with axial symmetry

$$
\begin{aligned}
g_{t t} & =\left(k-\frac{4 \beta m}{\rho r}\left(k \rho^{\beta}+\rho^{-\beta}\right)^{-1}\right) f^{2}, \\
g_{t r} & =\frac{\rho f}{2 \beta}\left(k \rho^{\beta}+\rho^{-\beta}\right), \\
g_{t x} & =\frac{x r f}{2 \rho \beta}\left[\left(k \rho^{\beta}-\rho^{-\beta}\right) \beta+k \rho^{\beta}+\rho^{-\beta}\right], \\
g_{t y} & =\frac{y r f}{2 \rho \beta}\left[\left(k \rho^{\beta}-\rho^{-\beta}\right) \beta+k \rho^{\beta}+\rho^{-\beta}\right], \\
g_{x x} & =g_{y y}=-r^{2}
\end{aligned}
$$

or

$$
g_{\mu \nu}=\left(\begin{array}{cccc}
-k^{2} r^{2} f^{2}+\frac{2}{f} f^{\prime}(t) r-\frac{2}{r} m f^{-3} & 1 & -k y r^{2} f^{2} & -k x r^{2} f^{2} \\
1 & 0 & 0 & 0 \\
-k y r^{2} f^{2} & 0 & -\rho^{2} r^{2} f^{2} & 0 \\
-k x r^{2} f^{2} & 0 & 0 & -\rho^{2} r^{2} f^{2}
\end{array}\right) \text {, }
$$


where $k, m, \beta$ are constants, $\rho=\sqrt{x^{2}+y^{2}}, f(t)$ is an arbitrary function, and $(t, r, x, y)$ are the coordinates.

For cases without axial symmetry, we can get solutions as follows:

$$
g_{\mu \nu}=\left(\begin{array}{cccc}
4 k^{2}-r^{2} n^{2}-2 \frac{m}{k \cosh (2 x) r} & k \cosh (2 x) & 2 k \sinh (2 x) r & -n r^{2} \\
k \cosh (2 x) & 0 & 0 & 0 \\
2 k \sinh (2 x) r & 0 & -r^{2} & 0 \\
-n r^{2} & 0 & 0 & -r^{2}
\end{array}\right) \text {, }
$$

where $k, m, n$ are constants.

\section{Conclusion}

All the examples mentioned above always contain some black holes, i.e. singularities in Riemann tensor $R_{\mu \nu \alpha \beta}$, so they are not trivial solutions. These solutions show that the canonical metric is really convenient and powerful for solving the vacuum Einstein equation, and the procedure of resolution is quite straightforward.

There are many exact solutions collected in the books like[10, 11]. Most of them can be transformed into the canonical form (1.8). Some authors have realized the convenience of this kind of form related to the light cone, and the Robinson-Trautman type solutions are simple cases of (1.8). However these authors seem to pay attention mainly on using the advanced mathematical tools to certain special cases.

The main characters of our procedure is that:

(I) It presents a more general framework for getting exact solutions to the Einstein equation, which covers most of the known results for empty space.

(II) The procedure of resolution shows that the Einstein equation of (1.8) is an underdetermined system, so that we should take the components $a, b$ as given functions determined by coordinate conditions and boundary or initial conditions.

(III) When we take $a, b$ as given functions, the other components are decoupling variables in the system of equations in some sense, so we can use the recursive method to solve the Einstein equation.

(IV) The ansatz (2.3) or (2.24) is natural and necessary for getting exact solutions. As to the numerical solution, instead of this ansatz, we should input the appropriate values of $a, b$ as boundary or initial conditions to determine other components. 


\section{Acknowledgments}

The author is grateful to his supervisor Prof. Ta-Tsien Li for his encouragement and guidance. Thanks to Prof. S. Klainerman for suggesting me how to modify my original paper. Prof. Hao Wang helped me to effectively use the softwares.

\section{References}

[1] Matthias Stute, Max Camenzind, Towards a self-consistent relativistic model of the exterior gravitational field of rapidly rotating neutron stars, arXiv:astro$\mathrm{ph} / 0301466$

[2] Jiri, Bicak, Selected solutions of Einstein field equations: their role in general relativity and astrophysics, arXiv:gr-qc/0004016

[3] M.A.H. MacCallum, Finding and using exact solutions of the Einstein equations, arXiv:gr-qc/0601102v2

[4] Jiri, Bicak, Einstein equations: exact solutions, arXiv:gr-qc/0604102

[5] S. L. Weinberg, Gravitation and Cosmology(Ch.7.4, Ch.11.8), Wiley, New York, 1972

[6] Y. Q. Gu, A Canonical Form of the Metric in General Relativity, to appear

[7] C. Klein, O. Richter, Physical Realistic Solutions to the Ernst Equation on Hyperelliptic Riemann Surface, arXiv:gr-qc/9806051

[8] I. Hauser, F. J. Ernst, Group structure of the solution manifold of the hyperbolic Ernst equation, arXiv:gr-qc/9903104

[9] Y. Q. Gu, An Exact Solution of the Einstein Equation, arXiv:0705.2133v1 [gr-qc]

[10] D. Kramer, H. Stephani, E. Herlt, M. MacCallum, E. Schmutzer, Exact Solutions of Einstein's Field Equations, Cambridge Univ. Press 1980

[11] S. W. Hawking, G. F. R. Ellis, The Large Scale Structure of the Space-time, Cambridge Univ. Press 1999 